\title{
Valoración de habilidades gerenciales personales a empleados de organizaciones públicas y privadas, por parte de directivos
}

\section{Assessment of personal managerial skills to employees of public and private organizations, by managers}

\author{
Dolores Isabel Ramón Ramón \\ José Ollague Valarezo \\ Jaime Granda Bohórquez \\ Claudia Naranjo Sánchez \\ Universidad Técnica de Machala, Ecuador
}

Autor para correspondencia: diramon@utmachala.edu.ec,jollague@utmachala.edu.ec, jpgranda@utmachala.edu.ec, cenaranjo@utmachala.edu.ec

Fecha de recepción: 19 de Julio de 2017 - Fecha de aceptación: 10 de Agosto de 2017

Resumen: El presente trabajo contempla una investigación realizada a los empresarios de diversos sectores productivos de la provincia de El Oro, pertenecientes al sector público y privado. Su finalidad fue la valoración de las habilidades directivas personales que poseen sus colaboradores, para determinar si éstas son satisfactorias desde la percepción de los directivos. El desarrollo de habilidades personales ha ido cobrando cada vez mayor importancia, porque es a través de ellas que los colaboradores de una organización dan su aporte para lograr la competitividad empresarial, a través de su nivel de autoconocimiento y dominio de sí mismo, la administración del tiempo, su inteligencia emocional, la determinación de valores y prioridades y la solución creativa de problemas. Del resultado obtenido de la investigación, se comprobó que no existe una diferencia significativa entre la media de las habilidades: Nivel de autoconocimiento, Actuación en situaciones estresante y Solución analítica y creativa de problemas, en el recurso humano que se desenvuelve en las empresas públicas y privadas de la provincia de El Oro.

Palabras clave: habilidades gerenciales personales; recursos humanos; ventaja competitiva; organización; resultados

Abstract: The present work contemplates a research carried out to the entrepreneurs of diverse productive sectors of the province of the Gold, pertaining to the public and private sector. Its purpose was to assess the personal management skills of its employees, to determine if these are satisfactory from the perception of managers. The development of personal skills has become increasingly important, because it is through them that the collaborators of an organization give their contribution to achieve business competitiveness, through their level of self-knowledge and self-control, time, their emotional intelligence, the determination of values and priorities and the creative solution of problems. From the result obtained from the research, it was verified that there is no significant difference between the averages of the skills: Level of self-knowledge, Performance in stressful situations and Analytical and creative solution of problems, in the human resource that is developed in public companies and private companies in the province of El Oro.

Key words: personal management skills; human resources; competitive advantage; organization; results 


\section{Introducción}

El desafío que tienen las organizaciones actuales es ser competitivas, entendiéndose este concepto, como la capacidad de las empresas para entrar y consolidarse en mercados cada vez más exigentes; conduciendo eficazmente su gestión interna y externa, y posicionándose favorablemente hacia sus diversos públicos: el interno, que tiene una relación directa con la organización como son empleados, directivos, accionistas; su público externo compuesto de clientes, proveedores, asociados de negocios, bancos, organismos gubernamentales, la comunidad y la competencia. Toda organización busca como su máxima, la expansión y consolidación en el mercado, y gozar de superioridad en relación a sus competidores.

Los factores internos son los que más peso tienen en la competitividad de las organizaciones (Aragón Sánchez \& Rubio Bañón, 2005), uno de los factores clave son los recursos humanos. Esta dinámica empresarial impone a las organizaciones disponer de recurso humano con habilidades gerenciales desarrolladas, debido a que es a través de ellos que se hace efectiva la estrategia organizacional. (Pereda Pérez, López-Guzmán, \& González Santa Cruz, 2014), refieren que en la actualidad las habilidades personales, interpersonales y directivas, se convierten en ventaja competitiva para las empresas, debido a que es el recurso humano el que lidera los procesos de mejora.

Las organizaciones deben responder al mercado de manera oportuna y eficaz, con mayor efectividad que su competencia, centrando sus esfuerzos en optimizar sus recursos, productividad y rentabilidad, condición que es factible a través de las habilidades gerenciales que tengan sus directivos y colaboradores.

Las habilidades gerenciales o directivas, son definidas por (Whetten \& Cameron, 2011), como el conjunto identificable de acciones que los individuos llevan a cabo y conducen a ciertos resultados que aseguran el éxito organizacional. Si las empresas desean tener éxito deben contar con directivos hábiles y competentes, y desarrollar al máximo su potencialidad. Las empresas requieren gerentes con múltiples conocimientos y una amplia gama de habilidades y con la capacidad de liderar grupos altamente capacitados. (Naranjo Arango, 2012)

Otra definición formulada por (Sánchez, 2012), expresa que las habilidades gerenciales son el conjunto de capacidades y conocimientos que posee una persona y que son fundamentales para la dirección de una empresa, las empresas deben su éxito o fracaso a la acertada conducción de la misma. Las habilidades gerenciales con consideradas además, como fuente de ventaja competitiva para impulsar el rendimiento de la empresa, el capital humano aporta creación de valor (Datta \& Iskandar-Datta, 2014).

De acuerdo a la Real Academia de la Lengua, el concepto de habilidad proviene del término latino habilitas y es el talento o la capacidad para desarrollar una actividad de manera idónea. La palabra gerencia procede del latín genere, que se la identifica como administrar, relacionando estos dos términos, las habilidades gerenciales son el conjunto de conocimientos, aptitudes y destrezas que debe poseer el recurso humano de una organización. Las habilidades gerenciales propuestas por (Whetten \& Cameron, 2011), se componen de habilidades interpersonales para el manejo de conflictos, motivación de los empleados, comunicación de apoyo y ganar poder e influencia. Las habilidades grupales están conformadas por la formación 
de equipos efectivos, liderar el cambio positivo y el facultamiento y delegación.

Complementarias a estas, se encuentran otras habilidades que son fundamentales, las habilidades gerenciales personales, que gobiernan el comportamiento propio, incorporando efectividad en las acciones individuales de cada empleado. Si se carece de las mismas o ha habido un limitado desarrollo, su desempeño será deficiente, con escaso impacto en el logro de resultados para la empresa. Las habilidades directivas personales abarcan el desarrollo del autoconocimiento, el manejo del estrés personal y la solución creativa de problemas.

El desarrollo de habilidades personales ha cobrado gran importancia en los últimos tiempos, debido al aporte que realiza el empleado para su organización a través de su actuación con inteligencia emocional, administrando eficazmente el tiempo y solucionando de manera creativa los problemas de la organización, para alcanzar objetivos estratégicos y conducir su actuación personal con asertividad, estableciendo prioridades, conociéndose a sí mismo para que su aporte individual tenga efectividad y le agregue competitividad a la organización. Es la capacidad y el conocimiento que una persona posee para ejercer el liderazgo y hacer que los miembros de una organización contribuyan para su éxito.

Dentro de las habilidades gerenciales personales, es fundamental el desarrollo del autoconocimiento, para poder conducirse a sí mismo y dirigir a otros y alcanzar resultados satisfactorios. Se debe desarrollar y perfeccionar habilidades que le faciliten el conocerse de manera particular, sus motivaciones, sentimientos, necesidades, identificar las situaciones que son causas de estrés para ejercer control sobre ellas, manejo de autocontrol, control mental para encauzar las situaciones negativas que se dan en el campo laboral.

En el desarrollo del autoconocimiento debe cuestionarse, que tanto un individuo se conoce a sí mismo, cómo responde a los estímulos de su entorno, cómo es su autoaprendizaje. De acuerdo a lo expresado por (Whetten \& Cameron, 2011), es poder conocerse a sí mismo, para lograr un autodominio o auto control y tener claras las prioridades y las metas. Sócrates promovía el conocerse a sí mismo, como indicio de sabiduría y autodominio.

Para lograr un autoconocimiento se precisa identificar las fortalezas personales que se posee y fortalecerlas constantemente, e igualmente detectar debilidades, para trazar un plan de acción para superarlas (Martínez, Hernández, \& Gómora, 2016).

Ligado al desarrollo del autoconocimiento está la inteligencia emocional, que expresado por (Goleman, 2012), es la capacidad de sentir, entender controlar y modificar los estados emocionales en uno mismo y en los demás, debiendo equilibrarse las emociones. La inteligencia emocional es la capacidad para conocer y controlar emociones propias y ajenas con el fin de obtener determinados fines. Este factor es de gran importancia, según psicólogos de renombre mundial, para que un individuo tenga éxito en su accionar su coeficiente intelectual contribuye solo con el $20 \%$, el $80 \%$ depende de su inteligencia emocional.

La inteligencia emocional es esencial para que los empleados puedan conseguir metas trazadas, son ellos los que le dan el valor agregado a una marca haciendo tangible lo intangible, a través de las competencias emocionales que desarrollen, no solo sabrán conducir sus emociones propias, sino gestionar las emociones de los demás, obteniendo lo mejor de ellos para 
conseguir resultados en bien de la organización. Tener inteligencia emocional es mejorar la comunicación, desarrollar empatía, generar confianza, crear un vínculo personal y duradero. El manejo propio es la habilidad fundamental de las habilidades gerenciales.

Otro componente es el manejo del estrés personal que según (Van-der Hofstadt \& Gómez, 2013), es el proceso que se inicia ante un conjunto de demandas ambientales que recibe el individuo, a las que debe dar una respuesta adecuada y oportuna, cuando la demanda es excesiva frente a los recursos para responder a esa demanda, se produce una activación a nivel fisiológico y reacciones emocionales como la ansiedad, la ira y la depresión.

La (Oficina Internacional del Trabajo -OIT-, 2016) revela que el impacto del estrés en la productividad y los costos económicos se evidencian en mayor absentismo y presentismo, menor motivación, satisfacción y compromiso; rotación del personal e intención de renunciar y un rendimiento reducido (menor eficiencia y precisión). Refiere que estos problemas afectan a la productividad, la competitividad y la imagen pública de la empresa, lo que es desfavorable para la organización en términos financieros y de prestigio.

Según (García-Campayo, 2015) El estrés debe ser entendido como un problema interactivo que involucra tanto al trabajador como a la organización, es un detonante de diversas enfermedades físicas, psicológicas, emocionales, hostilidad, que traen como consecuencia el síndrome de burnout o agotamiento del trabajador.

Como prevención del estrés en el trabajo la (Oficina Internacional del Trabajo, 2013), recomienda, un adecuado entorno físico y un equilibrio en el trabajo-vida y tiempo de permanencia en el trabajo, planificación del trabajo para evitar exigencias innecesarias y la subutilización de las capacidades de los trabajadores.

En lo relacionado a la administración del tiempo, los empleados dedican tiempo a múltiples actividades que no aportan a los objetivos prioritarios individuales y de la organización. Cuando no existe la capacidad para organizar el trabajo, se dejan asuntos pendientes, se duplican las operaciones, se hacen procesos repetitivos, existe desgaste físico y emocional innecesario debido al estrés de las actividades acumuladas, esto conlleva a un sentido de culpa y frustración porque no se alcanzan resultados satisfactorios, debido a la ineficiente planificación de actividades. No se sigue un curso de acción que contribuya a logros esperados por la organización y prevalecen las actividades postergadas.

Por otra parte, las organizaciones requieren de colaboradores que tenga la capacidad para dar una solución analítica y creativa de problemas, para (Mengua, Sempere, Juárez, \& Rodríguez, 2012) la actividad directiva exige el desarrollo de esa capacidad para definir con claridad los problemas, dar soluciones múltiples y diversas, evaluar las soluciones dadas y seleccionar las idóneas en cada situación, aplicando el pensamiento lógico y el creativo y además de la aplicación de técnicas para el desarrollo de soluciones.

El contar con habilidades hace la diferencia en el desempeño en el trabajo, que según (Judge \& Kammeyer-Mueller, 2012), el comportamiento de los empleados que son consistentes con expectativas de rol contribuyen a la eficacia con su actuación, desarrollan un liderazgo, que 
de acuerdo a lo descrito por (Rego, Vitória, Magalhaes, Ribeiro, \& Pina, 2013), es un patrón de comportamiento basado en valores, altos niveles de autenticidad, que promueve las capacidades psicológicas positivas y un clima ético positivo, potenciando al equipo para un compromiso afectivo con la organización.

Por consiguiente, los empleados necesitan capacidades específicas para responder a los muchos requerimientos de la organización, por tanto los niveles de habilidad de los empleados deben actualizarse constantemente para que se desempeñen con efectividad. (Detsimas, Coffrey, \& Zadiqui, 2016).

Es la suma de habilidades de directivos y colaboradores las que conforman una organización competitiva, con compromiso organizacional, con apego afectivo, con sentimientos de lealtad hacia ella. Es la contribución de las habilidades de directivos y colaboradores que conforma una organización competitiva, por lo que al evidenciarse un escaso desarrollo de las habilidades directivas personales del recurso humano que conforma una organización, la empresa no tendrá ventaja competitiva ni sus resultados le serán satisfactorios.

A continuación se presenta una investigación acerca de las habilidades gerenciales personales de los empleados de organizaciones públicas y privadas de la provincia de El Oro, perteneciente al sector comercial, financiero, industrial y servicios públicos y privados.

El objeto del presente estudio es analizar la valoración de las habilidades directivas, desde los directivos a sus colaboradores, en empresas públicas y privadas. El estudio se centró en los directivos por cuanto al hacer que se auto valoren los empleados, en una muestra previa que se aplicó, éstos tenían tendencia a sobredimensionar sus habilidades personales, calificándolas como satisfactorias y muy satisfactorias, diluyéndose con ello la objetividad del estudio, por lo que se optó por indagar en los directivos de alta jerarquía y de mandos medios para conocer su percepción acerca de las habilidades gerenciales personales que poseen sus colaboradores que son reveladas en su desempeño diario.

Al determinar el grado de percepción que tienen los directivos sobre determinadas habilidades de sus colaboradores, éstas servirán de referencia para que los empleados puedan desarrollar sus habilidades en los aspectos donde muestran deficiencias focalizadas.

\section{Materiales y Métodos}

La investigación fue llevada a cabo mediante un estudio empírico centrado en empresas del sector gubernamental y empresas de varios sectores productivos de la provincia de El Oro. El enfoque utilizado fue del tipo cuantitativo y el método escogido el deductivo lógico. El diseño utilizado fue el no experimental del tipo transeccional descriptivo. El instrumento de medición empleado para la obtención de la información fue una encuesta estructurada en tres bloques de veinte y tres preguntas de acuerdo a la clasificación propuesta por Whetten \& Cameron (2011).

Para el análisis estadístico descriptivo de los datos se utilizó el programa SPSS versión 24.00, así mismo se aplicó el test de Kolmogorov-Smirnov y la prueba de t-Student. Para realizar 
el análisis factorial confirmatorio (AFC) se usó el software AMOS versión 22.00 donde se aplicó el método de máxima verosimilitud.

\section{Fiabilidad de los instrumentos}

Para determinar la fiabilidad y la validez de las diferentes escalas (tipo Likert), se utilizó el alfa de Cronbach, puesto que la medida de fiabilidad asume que los ítems miden el mismo constructo y están altamente relacionados (Welch y Comer, 1988). Para evaluar los coeficientes se tomó como recomendación los valores propuestos por George \& Mallerly (2003) con un coeficiente de alfa de $>0.60$.

\section{Procedimientos de muestreo}

El plan de levantamiento de la información se realizó en base a la información proporcionada por el Instituto Nacional de Estadísticas y Censos quien ha creado un registro empresarial que toma consideración la información de dos fuentes: el Servicio de Rentas Internas y la Superintendencia de compañías.

El concepto que subyace en el Directorio de Empresas mantiene una estructura conceptual de tres unidades propuestas por la Comunidad Andina de Naciones (CAN) y especificada en su Manual sobre Directorio de Empresas con Fines Estadísticos (2010). Para el efecto, una unidad legal son unidades legales son aquellas que la ley reconoce el derecho a tener un nombre propio o patrimonio, de establecer contrato con terceros y defender sus intereses frente a tribunales. En este mismo sentido una Empresa es una unidad organizativa que realiza actividades económicas en un uno o varios lugares. En cambio un establecimiento o unidad local, es la parte de una empresa ubicada en un lugar delimitado topográficamente, de donde la empresa ejerce sus actividades productivas (CEPAL, 2003). Para el presente estudio se considera que una unidad legal es responsable de una empresa y cada empresa ejerce su actividad de una unidad local.

\section{Tamaño de la muestra}

Para las organizaciones públicas el universo de estudio se ubica en 113 instituciones registradas en el Ministerio de gobierno. En cambio para las organizaciones privadas el universo de estudio lo componen todas las sociedades o empresas (562) abiertas en la Ciudad de Machala de la provincia de El Oro, cuya actividad éste relacionada a la venta de productos o servicios. Se descartan las sociedades cerradas (271).

Tabla 1 Resumen de organizaciones encuestadas

\begin{tabular}{llll}
\hline Descripción & Universo & Encuestadas & $\%$ \\
\hline Organizaciones publicas & 113 & 51 & $45,13 \%$ \\
Organizaciones privadas & 562 & 127 & $22.59 \%$ \\
Totales- $\rightarrow$ & 400 & 178 & --------- \\
\hline
\end{tabular}

Fuente: https: //sri.gob.ec, https://supercias.gob.ec

https://view.officeapps.live.com/op/view.aspx?src=http://descargas.sri.gov.ec/download/excel/listado_publicas_5_0 5_05.xls

\section{Medidas}


Para medir las variables se utilizó una escala de Likert de 1 a 5, de completamente en desacuerdo hasta completamente de acuerdo. La aplicación del instrumento de medición consistió en una adaptación de la encuesta PAMS (Personal Assessment of Management Skills) del libro de Desarrollo de habilidades directivas de Whetten \& Cameron (2011).

Tomando en consideración que la encuesta original tuvo una proporción de observaciones de ítems de 59.5 (5000/84 ítems) el presente trabajo mantiene una proporción de 7.73 (178 /23 ítems), lo cual es concordante con los valores que sugiere Aldas \& Maldonado (2004) para quienes se deben tener por lo menos cinco veces más observaciones que variables a ser observadas. Para la agrupación de los ítems se coincide con los autores de la herramienta original.

Las dimensiones de habilidades personales, contiene los factores Desarrollo de autonocimiento (AN), 5 ítems; actuación en situaciones estresantes (ASEBPT), 7 ítems y solución analítica y creativa de problemas(SACP), 8 ítems.

Las unidades de análisis se observaron naturalmente sin ninguna intervención por parte del equipo investigador, se utilizó el envío del formulario vía electrónica a todo el universo de estudio y luego de esto se estructuro una base de datos con aquellas instituciones que no contenían datos perdidos ni valores atípicos.

\section{Resultados}

Los resultados encontrados para los diferentes factores se pueden apreciar en la Tabla $\mathrm{N}^{\circ}$ 2, las habilidades personales está compuesta por tres factores: nivel de autoconocimiento, actuación en situaciones estresante y solución analítica y creativa de problema. Tal como se aprecia los coeficientes de confiabilidad se mantienen en valores cercanos a lo recomendado, mientras que la varianza media extraída mantiene este misma situación.

Tabla 2 Análisis de la validez convergente y fiabilidad de la capacidad Adaptativa

\begin{tabular}{ccccc}
\hline Variables latentes & $\begin{array}{c}\text { Siglas de } \\
\text { variables } \\
\text { observadas }\end{array}$ & $\begin{array}{c}\text { Parámetros } \\
\text { estandarizados }>0,5\end{array}$ & $\begin{array}{c}\text { Coeficiente } \\
\text { Compuesto de } \\
\text { Fiabilidad }>0,7\end{array}$ & $\begin{array}{c}\text { Varianza Media } \\
\text { Extraída (AVE) } \\
>0,5\end{array}$ \\
\hline $\begin{array}{c}\text { Nivel de } \\
\text { autoconocimiento }\end{array}$ & NA3 & 0,633 & 0,557 & 0,429 \\
NA4 & 0,778 & & \\
Actuación en & NA7 & 0,53 & 0,633 & 0,434 \\
situaciones & & & & \\
estresante & ASEBPT9 & 0,582 & & \\
& ASEBPT11 & 0.63 & & \\
& ASEBPT14 & 0.661 & & \\
& & & & \\
\end{tabular}




\begin{tabular}{ccccc}
\hline $\begin{array}{c}\text { Solución analítica } \\
\text { y creativa de } \\
\text { problema }\end{array}$ & ASEBPT15 & 0,751 & 0.617 & 0,405 \\
& & & & \\
& SACP16 & 0.585 & \\
& SACP17 & 0.657 & \\
& SACP20 & 0,696 & \\
& SACP21 & 0,600 & \\
\hline
\end{tabular}

Para la validez discriminante se utilizó el test de la varianza extraída, el procedimiento consiste en comparar la varianza extraída en cada factor y ésta se coloca en la diagonal principal, mientras que, en las celdas de los respectivos cruces, se colocan las correlaciones al cuadrado. . El criterio de validez discriminante (Tabla 3), es: si la correlación al cuadrado es mayor que la varianza extraída se puede afirmar que no hay validez discriminante, en caso contrario, se puede afirmar que los factores involucrados si discriminan y es lo recomendado. Por lo tanto se puede deducir que no se cumple con la validez convergente para ninguna de las dimensiones propuestas.

Tabla 3 Análisis de la validez discriminante para la capacidad de Aprendizaje

\begin{tabular}{llll}
\hline Variables latentes & $\begin{array}{l}\text { Nivel de } \\
\text { autoconocimiento }\end{array}$ & $\begin{array}{l}\text { Actuación en } \\
\text { situaciones } \\
\text { estresante }\end{array}$ & $\begin{array}{l}\text { Solución analítica } \\
\text { y creativa de } \\
\text { problema }\end{array}$ \\
\hline $\begin{array}{l}\text { Nivel de } \\
\text { autoconocimiento }\end{array}$ & 0,429 & 0,840 & 0,640 \\
$\begin{array}{l}\text { Actuación en situaciones } \\
\text { estresante }\end{array}$ & 0,434 & 0,816 \\
$\begin{array}{l}\text { Solución analítica y } \\
\text { creativa de problema }\end{array}$ & & 0,405 \\
\hline
\end{tabular}

Tal como se aprecia en la Tabla 4 las dimensiones donde valoraciones de las organizaciones privadas son mayores, son en Nivel de autonocimiento y Solución analítica y creativa de problema, mientras que los valores donde las organizaciones públicas tienen valores superiores a los de las organizaciones privadas se encuentran en el factor de Actuación en situaciones estresantes.

Tabla 4 Resumen de estadística por tipo de sociedad

\begin{tabular}{cccccc}
\hline Descripción & $\begin{array}{c}\text { Tipo de } \\
\text { sociedad }\end{array}$ & $\mathrm{N}$ & Media & $\begin{array}{c}\text { Desviación } \\
\text { estándar }\end{array}$ & $\begin{array}{c}\text { Media de } \\
\text { error estándar }\end{array}$ \\
\hline Nivel de & Privadas & 127 & 31,22 & 4,534 &, 402 \\
autoconocimiento & Publicas & 51 & 30,55 & 5,866 &, 821 \\
Actuación en situaciones & Privadas & 127 & 29,81 & 4,231 &, 375 \\
estresante & Publicas & 51 & 30,67 & 4,389 &, 615 \\
Solución analítica y & Privadas & 127 & 31,07 & 4,063 &, 361 \\
creativa de problema & Publicas & 51 & 32,25 & 3,989 &, 559 \\
\hline
\end{tabular}

Fuente: Base de datos 
En promedio las valoraciones de los tres factores se ubican mayoritariamente en las puntuaciones de acuerdo (4) y completamente de acuerdo (5), según la escala de Likert propuesta. En promedio más del $95 \%$ de todas las puntuaciones encajan en las dos opciones más altas de la escala. Lo cual ocurre tanto para organizaciones privadas como públicas, sin ningún distingo.

Tabla 5 Resumen de estadística por tipo de sociedad sobre habilidades (\%)

\begin{tabular}{ccccccc}
\hline Descripción & $\begin{array}{c}\text { Tipo de } \\
\text { sociedad }\end{array}$ & $\begin{array}{c}\text { Completamente } \\
\text { en desacuerdo }\end{array}$ & $\begin{array}{c}\text { En } \\
\text { desacuerdo }\end{array}$ & $\begin{array}{c}\text { Ligeramente } \\
\text { en } \\
\text { desacuerdo }\end{array}$ & $\begin{array}{c}\text { De } \\
\text { acuerdo }\end{array}$ & $\begin{array}{c}\text { Completamente } \\
\text { de acuerdo }\end{array}$ \\
\hline $\begin{array}{c}\text { Nivel de } \\
\text { autoconocimiento } \\
\text { Actuación en } \\
\text { situaciones }\end{array}$ & $\begin{array}{c}\text { Privadas } \\
\text { Publicas }\end{array}$ & $0.00 \%$ & $1.57 \%$ & $6.30 \%$ & $57.48 \%$ & $34.65 \%$ \\
$\begin{array}{c}\text { estresante } \\
\text { Solución }\end{array}$ & Publicas & $0.00 \%$ & $3.92 \%$ & $3.92 \%$ & $58.82 \%$ & $33.33 \%$ \\
$\begin{array}{c}\text { analítica y } \\
\text { creativa de } \\
\text { problema }\end{array}$ & Publicas & $0.00 \%$ & $0.00 \%$ & $3.15 \%$ & $31.50 \%$ & $65.35 \%$ \\
& & $0.00 \%$ & $1.96 \%$ & $0.00 \%$ & $25.49 \%$ & $72.55 \%$ \\
\end{tabular}

Fuente: Base de datos

Tabla 6 Prueba de normalidad

\begin{tabular}{lllll}
\hline \multirow{2}{*}{ Descripción } & $\begin{array}{l}\text { Tipo de } \\
\text { sociedad }\end{array}$ & \multicolumn{2}{l}{ Kolmogorov - Smirnov } \\
& Estadístico & gl & Sig. \\
\hline Nivel de & Privadas &, 166 & 127 &, 000 \\
autoconocimiento & Publicas &, 119 & 51 &, 069 \\
Actuación en situaciones & Privadas &, 098 & 127 &, 005 \\
estresante & Publicas &, 106 & 51 &, 200 \\
$\begin{array}{l}\text { Solución analítica y } \\
\text { creativa de problema }\end{array}$ & Privadas &, 097 & 127 &, 005 \\
\hline
\end{tabular}

Esto es un límite inferior de la significación verdadera. Corrección de significación de Lilliefors

Para corroborar la normalidad de la variable aleatoria que en ambos grupos se distribuye normalmente, se utilizó la prueba Kolmogorov-Smirnov (K-S) que se utiliza para muestras grandes (>30). El criterio para determinar si la (VA) se distribuye normalmente es: a) P-valor $\Rightarrow$ $\alpha$ Acepta $\mathrm{H} 0=$ Los datos provienen de una distribución normal; $\mathrm{y}, \mathrm{b}) \quad \mathrm{P}$-valor $<\alpha$ Acepta H1 = Los datos NO provienen de una distribución normal. Tal como se aprecia en la Tabla 6, el nivel de autonocimiento se comporta normalmente solo en las empresas públicas, no en las privadas por lo que se descarta la aplicación de la prueba t en este factor. En cambio, para las variables Actuación en situaciones estresante y Solución analítica y creativa de problema, se cumple con el criterio de normalidad.

Luego se ha proseguido con la aplicación de la prueba de Levenge bajo los supuestos de: a) $\mathrm{P}$-valor $=>\alpha$ Acepta $\mathrm{H} 0=$ Las varianzas son iguales y b) $\quad \mathrm{P}$-valor $<\alpha$ Acepta $\mathrm{H} 1=$ Existe diferencia significativa entre las varianzas, los resultados arrojan los siguientes valores: Pvalor (Nivel de autoconocimiento)= 075 ; P- valor (Actuación en situaciones estresante) $=, 893$; P- valor (Solución analítica y creativa de problema) $=, 741$, por lo que se deduce que existe igualdad entre las varianzas para todas las variables analizadas. 
Finalmente, los resultados del Análisis Factorial Confirmatorio y el método de máxima verosimilitud en el software AMOS 23, confirma que los constructos Nivel de autoconocimiento, Actuación en situaciones estresante y Solución analítica y creativa de problema tuvieron problemas de estructura, por lo que se tuvo que realizar ajustes al modelo original propuesto en la Figura 1.

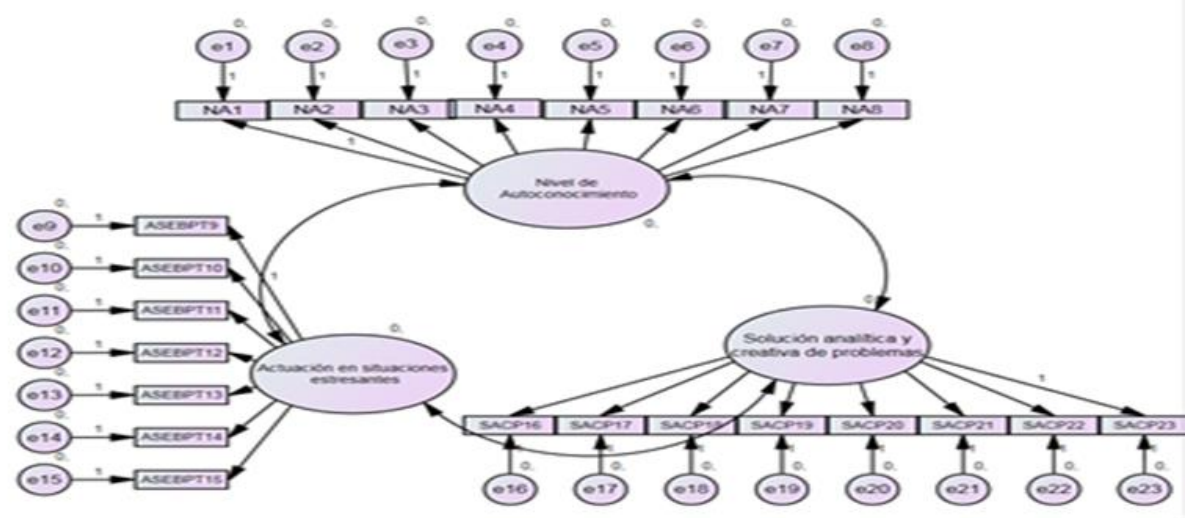

Figura 1. Modelo de estructura

Las variables NA4, ASEBPT15 y SACP20 superaron el nivel de fiabilidad según los valores sugeridos por Nunnally \& Bernstein (1994) y Hair et al (1995). El índice de la varianza extraída (IVE) en todas los factores es inferior al 0.5, según los valores sugeridos por Fornell \& Larcker, sin embargo este nivel no se encuentra tal alejado del umbral mínimo propuesto, además es fácil encontrar en la literatura ejemplos de escalas aceptadas con IVE inferiores Aldás \& Maldonado (2004).

El modelo final (Figura 2) sugiere que el modelo proporciona un buen ajuste Chi cuadrado de 55.928 con 41 grados de libertad y un $60 \%$ de nivel de probabilidad, lo cual a pesar de que los indicadores globales no son los mejores existen otros indicadores que nos permiten llegar a un modelo matemático sobre identificado $(\mathrm{NFI}=0.908 ; \mathrm{CFI}=0.973 ; \mathrm{FMIN}=0.316 ; \mathrm{y}$ RMSEA =0.045), según los valores mínimos propuestos por Wheaton et al (1977), Byrne (2010) y Bentler (1988).

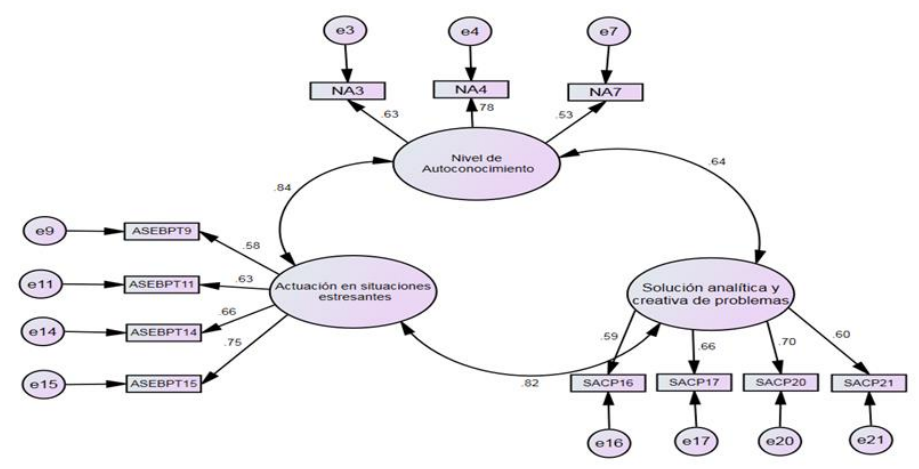

Figura 2. Modelo final ajustado

Reclutamiento 
El procedimiento para la recolección de la información consistió en el envío de un cuestionario vía electrónica en el periodo comprendido desde septiembre/2016 a febrero/2017, en este caso los periodos de contratación y seguimiento coinciden tanto para las organizaciones públicas como las privadas.

\section{Conclusión}

La medición de las habilidades nos permitió tomar como recomendación que es necesario mejorar los niveles de confiabilidad para un próximo estudio de tal forma que los coeficientes no se encuentren en los umbrales de aceptación, sino que se manejen con valores de alta confiabilidad. Para el caso del método de la validez convergente se aplica la misma recomendación al igual que el método de máxima verosimilitud en el Análisis Factorial Confirmatorio. Para normalidad, es importante aclarar que tan solo la variable Nivel de autoconomiento no se comportó normalmente para ambos grupos, para las demás comparaciones las mediciones fueron positivas.

El presente estudio concluye que no existe una diferencia significativa entre la media de las habilidades: Nivel de autoconocimiento, Actuación en situaciones estresante y Solución analítica y creativa de problemas, en el capital humano que se desenvuelve en las empresas públicas y privadas de la provincia de El Oro. Por otra parte, varios autores (Lombana, 2014), (Aburto \& Bonales, 2011); (Arroyo, 2012); coinciden en que estas habilidades son fundamentales para un óptimo desempeño organizacional, debido a que las organizaciones precisan no solo de recurso humano con sólida preparación técnica, sino que sepa manejarse a sí mismo con efectividad en ambientes exigentes, de mucha presión por alcanzar metas empresariales.

No le hace mucho favor a una empresa tener un técnico destacado en una determinada materia, pero con limitadas habilidades personales, (Cuadra \& Veloso, 2007) porque no logrará empatía, cohesión con los miembros de la organización, participación en el trabajo en equipo, lo que dificultará que la organización alcance metas comunes planteadas, y por otra parte que el ambiente laboral se torne inapropiado, (Salazar Estrada \& al., 2009) trayendo como consecuencia el desgaste del recurso humano o "bornout", (Ramírez \& Sau-Lyn, 2011), lo que no es conveniente para las organizaciones porque estas situaciones provocan que su capital humano no de lo máximo de sí mismo en beneficio de la organización y esto le resta competitividad en el mercado.

\section{Agradecimientos}

A los empleados de empresas públicas y privadas que colaboraron proporcionando la información relacionada con sus superiores. Se hace extensivo el agradecimiento a la revista Innova Journal por la apertura para la publicación del presente manuscrito. Se expresa el imperecedero agradecimiento a las empresas públicas y privadas de los diversos sectores productivos de la provincia de El Oro, que participaron en la presente investigación con sus oportunas respuestas y valiosos comentarios.

\section{Bibliografía}


Aragón Sánchez, A., \& Rubio Bañón, A. (2005). Factores asociados con el éxito competitivo de las pymes industriales en España. Universia Business Review, 38-51.

Datta, S., \& Iskandar-Datta, M. (2014). Upper-Echelon Executive Human Capital. Strategic Management Journal, 14.

Detsimas, N., Coffrey, V., \& Zadiqui, Z. (2016). Workplace Training \& Generic and Technical Skill. Journal of Management Development, 19.

García-Campayo, e. a. (2015). Dermatology Burnout Syndrome and Demotivation among HealthCare Personnel. Managing Stressful Situations: TheImportance of Teamwork. Actas Dermo-Sifiolográficas, 1-7.

Goleman, D. (2012). Inteligencia emocional. Barcelona: Kairós.

Judge, T. A., \& Kammeyer-Mueller, J. D. (2012). Job Attitudes. Annual Reviews, 341-367.

Kor, Y., \& Mesko, A. (2012). Dynamic managerial capabilities: configuration and orchestration of top executives'capabilities and the firm's dominant logic. Strategic Management Journal, $1-12$.

Martínez, M., Hernández, M., \& Gómora, J. (2016). Modelo de competencias directivas en escenarios globales para las instituciones de educación superior. Revista Iberoamericana para la investigación y el desarrollo educativo, 1-13.

Mengua, A., Sempere, F., Juárez, D., \& Rodríguez, A. (2012). La resolución de problemas para la mejora continua y progreso de las empresas. Ciencias, Revista de Investigación, innovación y desarrollo, 1-22.

Naranjo Arango, R. (2012). Habilidades Gerenciales Del Líder En Las Medianas Empresas De La Región Caribe Colombiana. Teacs Revista científica "Teorías, enfoques y aplicaciones en las ciencias sociales", 26-36.

Oficina Internacional del Trabajo. (2013). La prevención del estrés en el trabajo: lista de puntos de comprobación. Mejores prácticas para la prevención del estrés en el lugar de trabajo. Turín: Centro Internacional de Formación de la OIT.

Oficina Internacional del Trabajo -OIT-. (2016). Estrés en el trabajo: un reto colectivo. Turín: Centro Internacional de Formación de la OIT.

Pereda Pérez, F., López-Guzmán, T., \& González Santa Cruz, F. (2014). Habilidades directivas como ventaja competitiva. El caso del sector público de la provincia de Córdova (España). Intangible capital, 528-561. 
Rego, A., Vitória, A., Magalhaes, A., Ribeiro, N., \& Pina, M. (2013). Are authentic leaders associated with more virtuos, committed and potent teamns? The Leadership Quarterly, 61-79.

Sánchez, J. (09 de 08 de 2012). Funciones, comptencias y habilidades directivas. Madrid, Madrid, España.

Van-der Hofstadt, C., \& Gómez, J. (2013). Competencias y habilidades profesionales para universitarios. Madrid: Ediciones Díaz de Santos.

Whetten, D. A., \& Cameron, K. S. (2011). Desarrollo de Habilidades Directivas. México: Pearson Educación.

\section{Apéndices}

\section{Tabla 7 Constructo de capacidad de Habilidades Gerenciales}

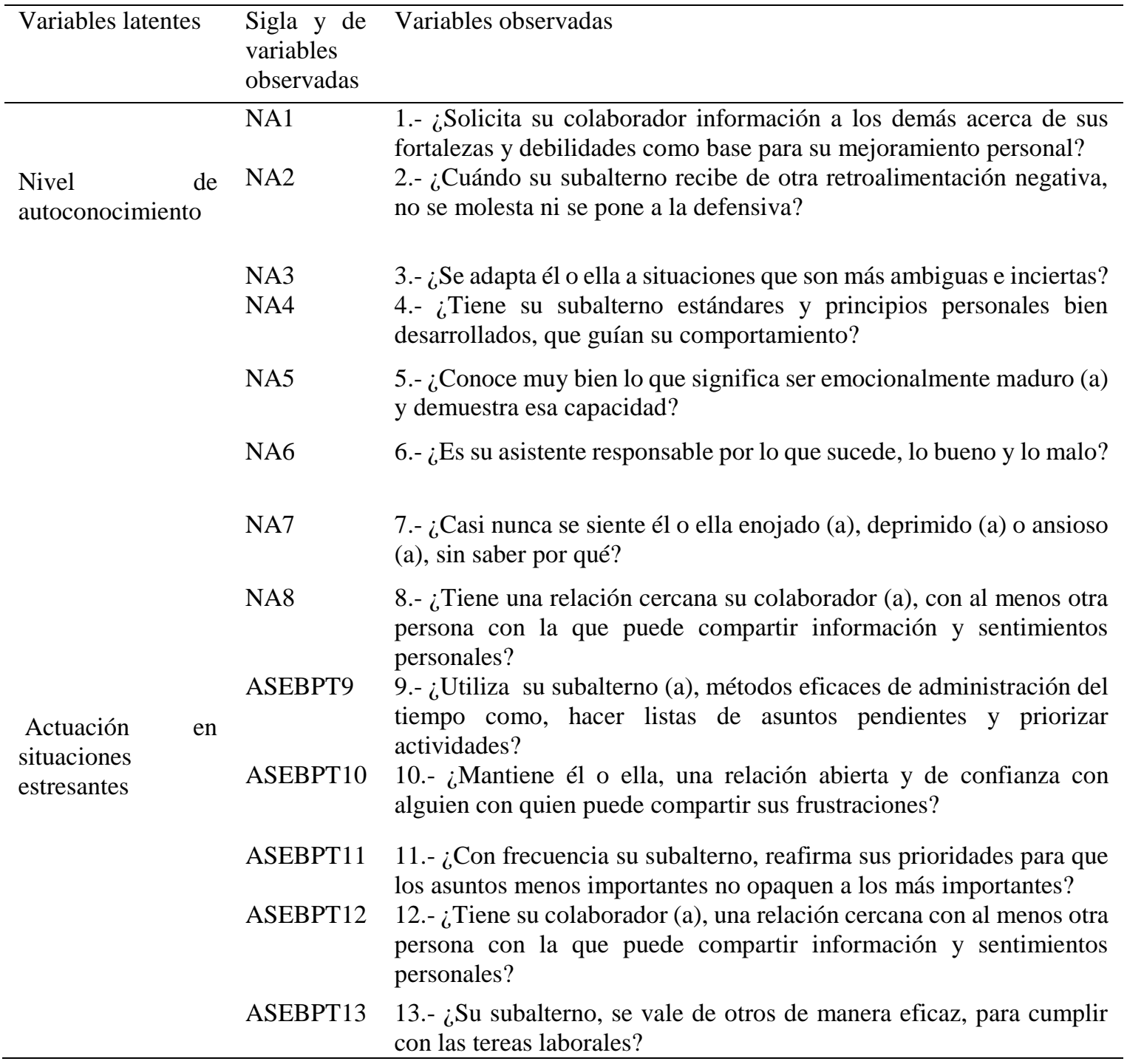




\begin{tabular}{|c|c|c|}
\hline \multirow{9}{*}{$\begin{array}{l}\text { Solución analítica } \\
\text { y creativa de } \\
\text { problemas }\end{array}$} & ASEBPT14 & $\begin{array}{l}\text { 14.- ¿Alienta su colaborador (a) los demás a generar soluciones } \\
\text { recomendadas? } \\
\text { 15.- ¿Lucha él o ella, por redefinir los problemas como oportunidades } \\
\text { para mejorar? }\end{array}$ \\
\hline & SACP16 & $\begin{array}{l}\text { 16.- ¿Cuando su asistente encuentra un problema de rutina, expresa de } \\
\text { manera clara y explícita cuál es el problema; o evita tratar de } \\
\text { resolverlo antes de haberlo definido? }\end{array}$ \\
\hline & SACP17 & $\begin{array}{l}\text { 17.- ¿Su colaborador, siempre genera más de una solución alternativa } \\
\text { al problema, en vez de generar solo una solución evidente? }\end{array}$ \\
\hline & SACP18 & $\begin{array}{l}\text { 18.- Su subalterno, se apega a las distintas etapas del proceso de } \\
\text { solución de problemas; esto es, define el problema antes de proponer } \\
\text { soluciones y genera alternativas antes de elegir una solución? }\end{array}$ \\
\hline & SACP19 & $\begin{array}{l}\text { 19.- Su colaborador, cuando se enfrenta a un problema difícil, ¿define } \\
\text { el problema en múltiples formas o se limita solo a una solución del } \\
\text { problema? }\end{array}$ \\
\hline & SACP20 & $\begin{array}{l}\text { 20.- ¿Trata su colaborador de ser flexible en la forma de enfrentar el } \\
\text { problema, al probar distintos métodos alternativos? }\end{array}$ \\
\hline & SACP21 & $\begin{array}{l}\text { 21.- ¿Cuenta él o ella, con técnicas específicas que utiliza para } \\
\text { desarrollar soluciones creativas e innovadoras a los problemas? }\end{array}$ \\
\hline & SACP22 & $\begin{array}{l}\text { 22.- ¿Trata su subalterno (a) de obtener información de individuos } \\
\text { ajenos al grupo para solucionar el problema, los cuales se verán } \\
\text { afectados por la decisión, para determinar sus preferencias y } \\
\text { expectativas? }\end{array}$ \\
\hline & SACP23 & $\begin{array}{l}\text { 23. ¿Alienta de manera informada el romper las reglas para obtener } \\
\text { soluciones creativas? }\end{array}$ \\
\hline
\end{tabular}

